\title{
Algorithms for Solving Inverse Problems of Simulation Modeling
}

\author{
EKATERINA GRIBANOVA \\ Tomsk State University of Control Systems and Radioelectronics, Lenina ave., 40, 634050, Tomsk, Russian Federation, (e-mail: geb@asu.tusur.ru)
}

Corresponding author: Ekaterina Gribanova (e-mail: geb@asu.tusur.ru).

\begin{abstract}
: ABSTRACT This paper is devoted to solving inverse problems of simulation modeling, which are presented in the form of an optimization problem. The article discusses the use of direct search methods taking into account the specifics of the problem under consideration. Due to the fact that these methods require a lot of computational experiments, two algorithms based on approximation were proposed for solving the problem. The first algorithm consists in determining and evaluating the parameters of the function of dependence (which can be linear or nonlinear) of the output variable on the input variables and solving the inverse problem by minimizing increments of the arguments. In the second algorithm a linear function of dependence is iteratively constructed using the data set generated by changing the input variables in given increments, and the inverse problem is solved by minimizing increments of the arguments. The classical inventory management model with a threshold strategy is considered as an example. The inverse problem was solved using direct search and approximation-based methods.
\end{abstract}

KEYWORDS inverse problems; simulation modeling; inverse calculations; mathematical optimization; function approximation; inventory control.

\section{INTRODUCTION}

CIMULATION modelling allows answering the Qquestion "What will happen, if...?" and it is a powerful tool for the study of complex systems. The behavior of the object under consideration is simulated closest to reality using the algorithm. So, this method is used in cases when the use of analytical methods is impossible due to the complexity of the studied phenomenon or when it is necessary to reproduce the dynamics of changes in the state of the object taking into account stochastic factors. Simulation modelling is used to study queuing systems, supply chains, production processes of enterprises, etc.

When making managerial decisions, experts need to answer the question "How to make it so that...?", i.e. they face the need to determine values of input variables, at which the specified indicators of object performance will be achieved [1-4]. Such problems emerged a long time ago, in particular, individual examples are given with reference to papers of ancient philosophers [5]. A big role in the study of such problems, called incorrect, and the development of the mathematical apparatus is played by such scientists as A.N. Tikhonov [1], M.M. Lavrentiev, V.K. Ivanov. It is often required to attract specialists and get expert judgments due to the complexity of inverse problems. So, B.E. Odintsov proposed an inverse computation apparatus for solving inverse problems in the field of economics stated in the following way: determining increments of function arguments based on their input values, the given function value, expert information which is the directions for changing the arguments, and relative priority coefficients [6]. The problem of finding a solution with a minimum change in the arguments [7], i.e. obtaining the solution closest to the initial one to get the given value of the resulting function, can be considered as a modification of such a problem. Here the input values characterize the current state of the object, therefore, their smaller change requires less effort to achieve the goal. In contrast to the problems of economic analysis considered in paper [6], where determinate dependence between arguments was considered, the dependence between the output variable and input 
variables has a stochastic form in simulation models. Therefore, one of the features of solving inverse problems of simulation modelling is that a given value may not be achieved during simulation, consequently, the inverse problem can be solved with some error. So, the problem can be presented as an optimization problem:

$$
|f-\widehat{f}| \rightarrow \min
$$

where $f$ - the given value of the output variable, $\hat{f}-$ the value of the output variable obtained in the course of simulation.

Gradient methods showed the greatest efficiency in solving optimization problems [8-11]. However, there is no analytical dependence function in simulation models; it is possible to obtain only discrete values of the output variable. Therefore, to solve the problem (1), the use of direct methods $[12,13]$ that are based on calculating the values of the output variable can be considered. However, their use may require much time and a lot of computing resources. The use of evolutionary algorithms showed high efficiency in solving global optimization problems [14, 15], however, their disadvantages include the complexity of their software implementation and the need to use a sufficiently large number of individuals and operations with them, and simulation is required for each calculation of the output variable. This may take a long time or require efforts to rework the model. So, in some tabular simulation models [16-18] changes in the input data of the model can lead to a change and addition of calculation formulas.

Among the existing solutions one can also mention ready-made programs for solving optimization problems of simulation modelling (for example, optquest), but some systems cannot be integrated with these products.

In this regard the urgent task is to develop an approach aimed at finding the solution to the inverse problem using fewer simulations as compared to direct search methods. One solution is to build a function of the relationship between the output variable and the input ones. The objective of this paper is to develop algorithms for solving the inverse problem based on function approximation followed by solving the inverse problem with the help of inverse computations while minimizing increments of the arguments.

\section{ALGORITHMS FOR SOLVING THE INVERSE PROBLEM OF SIMULATION MODELLING}

Let's consider the solution to the optimization problem (1) using direct search methods. The exploratory search method consists in changing the values of input variables in given increments. The following steps can be distinguished here:

Step 1. Determination of input values: input variables $x_{1}^{*}, x_{2}^{*}, \ldots, x_{m}^{*}, \widehat{x}_{i}=x_{i}^{*}(i=1 . . m)$, increment in changing input variables $\Delta x_{i}$, the preset value of the output variable $f$, the value of the output variable $\widehat{f}\left(f_{\min }^{*}=\widehat{f}\right)$ obtained in the course of simulation at the input data $x_{1}^{*}, x_{2}^{*}, \ldots, x_{m}^{*}$, the minimum increment $\varepsilon$ and the increment change coefficient $\gamma$.

Step 2. Performing an exploratory search around $x_{1}^{*}, x_{2}^{*}, \ldots, x_{m}^{*}$ by changing them by the increment value ( $\left.x_{i}=x_{i}^{*} \pm \Delta x_{i}\right)$ and determining the value of the output variable in the course of simulation of the output value $\hat{f}$.

Selection from $j$ options of the obtained sets of input values of the set that provides the minimum value of the difference between the preset value of the output variable and the value obtained in the course of simulation:

$$
\left|f-\widehat{f}_{j}\left(x_{1 j}, \ldots, x_{m j}\right)\right| \rightarrow \min .
$$

When solving the problem, the number of set $j$ and the corresponding values of the input and output variables will be determined $f_{\text {min }}\left(x_{1 \min }, \ldots, x_{m \text { min }}\right)$.

Step 3. Condition check: if the minimum value of the output variable is less than the best value of the output variable: $f_{\min }<f_{\min }^{*}$, then $f_{\min }^{*}=f_{\min }, \quad x_{i}^{*}=x_{i \min }$. Back to step 2.

Otherwise, if the increment value is less than the specified value: $\Delta x_{i} \leq \varepsilon$, then the algorithm ends.

Otherwise, the increment value is changed: $\Delta x_{i}=\Delta x_{i} \cdot \gamma$, and you go to step 2 .

The solution to the problem will be values $x_{i}^{*}$.

To increase the search efficiency, modifications of this method are used, which are based on the fact that in the case of finding the best solution, further research is carried out in the same direction. So, in case of adaptive search, at the second step changes in the arguments that led to a decrease in the value of the function are remembered and are used later in the next iteration. In case of the Hooke-Jeeves method [19], a movement is carried out in the direction that led to a decrease in the value of the function (pattern search).

The advantage of these methods is the simplicity of implementation; however, when using them, a sufficiently large number of iterations are required to find the solution. In this regard two algorithms based on approximation were developed.

The first algorithm is based on the construction of a function of the dependence of the output value in the input variables and includes the following steps:

Step 1. By performing simulations with different sets of input data, construct a function of the dependence of the output value on the input variables. The following three common dependencies in simulation models can be distinguished: 
1) linear;

2) nonlinear "with saturation": the output value is increased/decreased to a certain limit, after which the value of the variable remains almost at the same level (Fig.1a);

3) quadratic dependence (Fig.1b).

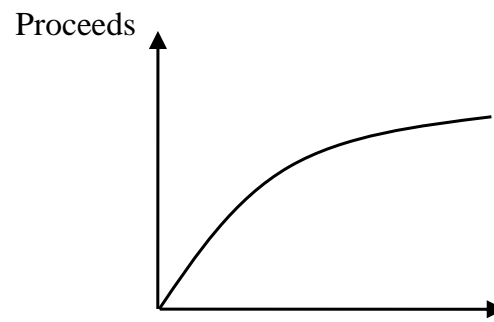

Number of

service channels

a)

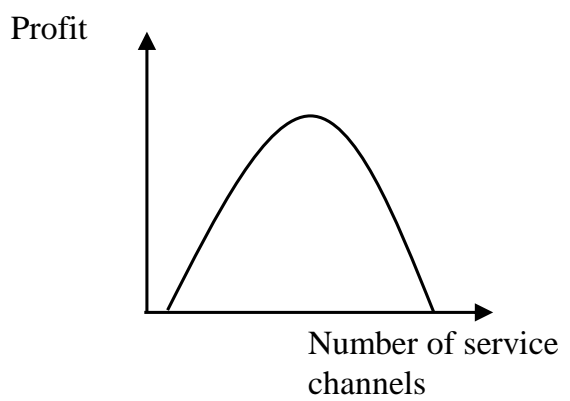

Figure 1. Types of dependencies: a) "with saturation"; b) quadratic

To estimate the parameters of the functions, the least square method can be used $(g(x)$ is the dependence function, $n$ is the number of observations) [20]:

$$
\sum_{j=1}^{n}\left(\widehat{f}_{j}-g\left(x_{j}\right)\right)^{2} \rightarrow \min
$$

Step 2. The inverse problem is solved using the function obtained in the first step. So, the problem looks as follows, in case of minimizing the increment of the function arguments:

$$
\begin{aligned}
& \sum_{i=1}^{m} \Delta x_{i}^{2} \rightarrow \min \\
& g(x+\Delta x)=f
\end{aligned}
$$

This problem refers to nonlinear optimization problems. To solve it, the penalization method, the Lagrange method of multipliers, etc. can be used. Paper [7] proposes to solve such a problem by solving a system of equations. Geometric construction was used to reveal that it is necessary to use numerical values for the arguments in the function $g(x)$ as the relations of the increments of the arguments to solve the problem with a linear function. If a nonlinear function is considered, then it can be determined through linearization that the numerical values at the arguments will be equal to the partial derivatives, thus, the ratios of the increments of the arguments are equal to the ratios of partial derivatives of the function $\left(x^{*}=x, k\right.$ is the number of the argument selected as the base one):

$$
\left\{\begin{array}{l}
\frac{\Delta x_{i}}{\Delta x_{k}}=-\frac{\beta_{i}}{\beta_{k}}, i=1 . . m, i \neq k \\
g\left(x_{1}+\Delta x_{1}, \ldots, x_{m}+\Delta x_{m}\right)=f .
\end{array},\right.
$$

$$
\text { where } \beta_{i}=\frac{\partial g\left(x^{*}\right)}{\partial x_{i}}, \beta_{k}=\frac{\partial g\left(x^{*}\right)}{\partial x_{k}} \text {. }
$$

In the case of the nonlinear function $g(x)$, the problem is solved iteratively: the values of the arguments modify on the basis of a given value of the parameter and a value of the partial derivative [7].

Step 3. Performing exploratory search using the values obtained in the previous step $x^{*}=x+\Delta x$ as the initial data.

Disadvantages of this method include the need to determine the type of dependence (linear, quadratic, etc.) and a sufficiently large set of data, and, therefore, simulations are needed to ensure high accuracy of approximation, which can take much time.

The second algorithm is based on linear approximation and solving the inverse problem (initial data: initial values of input variables $x_{1}^{*}, x_{2}^{*}, \ldots, x_{m}^{*}$, the preset value of the output variable $f$, the maximum absolute deviation from the preset value of the output variable $-\delta$ ):

Step 1. Formation of a set of input data for constructing the equation of dependence. Changes in input values $x_{1}^{*}, x_{2}^{*}, \ldots, x_{m}^{*}$ in increments of $\Delta x_{i}$, determination of the output variable $\widehat{f}$ in the course of simulation. Selection from the resulting set of elements $\widehat{x}_{1}^{*}, \widehat{x}_{2}^{*}, \ldots, \widehat{x}_{m}^{*}$ with the smallest absolute deviation of the output value from the given one $f$. The number of selected items shall be equal to the number of input variables. Determination of the element with the minimum deviation from the output $f$ (input variables $-x_{1 \min }^{*}, x_{2 \min }^{*}, \ldots, x_{m \text { min }}^{*}$, output variable $\left.f_{\min }^{*}\right)$ in the set.

Step 2. Construction of the equation of dependence of the output variable on the input values. The parameters $a$ can be found by the following formula using the least squares method: 


$$
a=\left(\widehat{x}^{* T} \widehat{x}^{*}\right)^{-1} \widehat{x}^{* T} \widehat{f}
$$

As a result, we will get the following equation:

$$
g(x)=a_{0}+\sum_{i=1}^{m} a_{i} x_{i}
$$

Step 3. Solution of the inverse problem: search for the amount of change in the arguments of the function to achieve the given value $f$. According to the previously obtained expressions, the increments are determined by the following formula:

$$
\Delta_{i}=\frac{-\left(a_{0}+a_{1} \cdot x_{1 \min }^{*}+\ldots+a_{m} \cdot x_{m \min }^{*}-f\right)}{\sum_{t=1}^{m} a_{m}^{2}} \cdot a_{i}
$$

Then the new values of the input variables are as follows: $x_{i}=x_{i \min }^{*}+\Delta_{i}$.

Step 4. Determination of the output variable $\widehat{f}$ at obtained $x_{i}$ in the course of simulation. Change of the values $x_{1}, x_{2}, \ldots, x_{m}$ in increments of $\Delta x_{i}$, determination of the output variable $\widehat{f}$ in the course of simulation. Selection from the resulting set of an element with the smallest deviation of the output value from the given $f$ (the value of the output variable is equal to $\widehat{f}_{\text {min }}$, of the input variables $\left.x_{1 \min }, x_{2 \min }, \ldots, x_{m \text { min }}\right)$.

If $\left|f-\widehat{f}_{\text {min }}\right|<\delta$, then the operation of the algorithm ends.

Otherwise if $\widehat{f}_{\min }>f_{\min }^{*}$, then it is required to conduct an exploratory search with respect to points $x_{1 \text { min }}^{*}, x_{2 \min }^{*}, \ldots, x_{m \text { min }}^{*}$. The algorithm ends.

Otherwise, assignment $x_{1}^{*}=x_{1 \min }, x_{2}^{*}=x_{2 \min }, \ldots, x_{m}^{*}=x_{m \text { min }}$ is performed, and then you go to step 1 .

When using the considered algorithms, it should be noted that it is necessary to perform a large number of random implementations when performing simulation, otherwise the results of solving inverse problems can differ significantly in different runs of the model.

The novelty of the proposed approach lies in the combination of the method of solving the inverse problems, shown in [7], with the exploration search and approximation, which allows solving the inverse problems of the simulation modeling, where output variables are of random nature.

\section{RESULTS OF COMPUTATIONAL EXPERIMENTS}

To conduct computational experiments, let's consider the classical inventory management model with a threshold strategy. To perform the experiments, Simulator system [21] was used. Input data:

1) initial level of inventory - 1000 pcs.;

2) average time of delivery of a batch of goods - 3 days; 1 day;

3 ) mean square deviation of the time of goods delivery -

4) average daily demand - 100 pcs;

5) mean square deviation of daily demand - 10 pcs;

$6)$ cost of storing an item of goods per unit of time - 2 monetary units;

7) cost of delivery of an item of goods - 50 monetary units;

8) deficiency penalty - 200 monetary units;

9) warehouse operation period - 30 days;

10) number of random implementations - 10,000 .

The objective of modelling is to determine the volume of the batch and the minimum level of stock ensuring the cost value equal to 200,000 monetary units. The initial values of the batch volume and the minimum stock level are 200 and 50, respectively. Parameter values for search algorithms: $\delta$ $=500, \varepsilon=25, \gamma=0.5$. We will use the Microsoft Excel package and the built-in VBA programming language to solve the inverse problem.

To form a function (a quadratic form was selected), values of the batch volume from 150 to 350 , values of the minimum stock level from 0 to 250 in increments of 50 were used in the first algorithm. The resulting function is as follows:

$$
\begin{aligned}
& g(x)=0.457 \cdot x_{1}^{2}+2.622 \cdot x_{2}^{2}+1.14 \cdot x_{1} x_{2}- \\
& -539.043 \cdot x_{1}-1604.43 \cdot x_{2}+462620.7
\end{aligned}
$$

where $x_{1}$-the volume of the batch, $x_{2}$ - the minimum stock level.

So, the inverse problem with a minimum change in the arguments of the function is as follows:

$$
\Delta x_{1}^{2}+\Delta x_{2}^{2} \rightarrow \min
$$

$$
\begin{aligned}
& 0.457 \cdot\left(200+\Delta x_{1}\right)^{2}+2.622 \cdot\left(50+\Delta x_{2}\right)^{2} \\
& +1.14 \cdot\left(200+\Delta x_{1}\right)\left(50+\Delta x_{2}\right)-539.043 \\
& \cdot\left(200+\Delta x_{1}\right)-1604.43 \cdot\left(50+\Delta x_{2}\right)+ \\
& +462620.7=200000 .
\end{aligned}
$$

Changes in the arguments during subsequent iterations (parameter $10^{-9}$ ) are presented in Fig.2. The result of problem solution: the batch volume is 243.89 , the minimum stock level is 192.98 . 


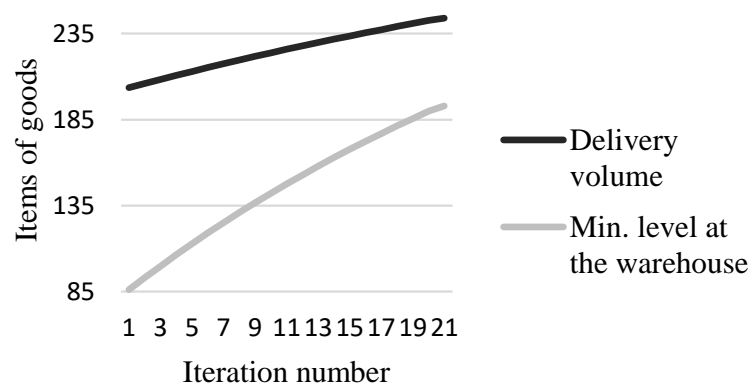

Figure 2. Changes in the function arguments when solving the inverse problem

Figure 3 shows the solution to the inverse problem of determining a set value of the function, while minimizing the sum of squares of changes of the arguments in the MathCad package. It may be concluded that the results obtained using the mathematical package and the proposed method are consistent (8).

The amount of the average cost obtained by simulation at given values of the batch volume and the minimum level of stock is 204799.2 monetary units.

Now consider the use of the second algorithm based on the construction of a linear dependence ( $\varepsilon=500$ ). Table 1 presents the results of simulation in the first step (the variables are changed in increments of 50).

$$
\begin{aligned}
& \mathrm{x} 1:=200 \quad \mathrm{x} 2:=50 \\
& \mathrm{f}(\Delta \mathrm{x} 1, \Delta \mathrm{x} 2):=(\Delta \mathrm{x} 1)^{2}+(\Delta \mathrm{x} 2)^{2}
\end{aligned}
$$

Given

$$
\begin{aligned}
& 0.457 \cdot(\mathrm{x} 1+\Delta \mathrm{x} 1)^{2}+2.622 \cdot(\mathrm{x} 2+\Delta \mathrm{x} 2)^{2} \ldots=200000 \\
& +1.14 \cdot(\mathrm{x} 1+\Delta \mathrm{x} 1) \cdot(\mathrm{x} 2+\Delta \mathrm{x} 2) \ldots \\
& +-539.043 \cdot(\mathrm{x} 1+\Delta \mathrm{x} 1)-1604.43 \cdot(\mathrm{x} 2+\Delta \mathrm{x} 2) \ldots \\
& +462620.7
\end{aligned}
$$$$
\text { res := Minimize }(\mathrm{f}, \Delta \mathrm{x} 1, \Delta \mathrm{x} 2)=\left(\begin{array}{c}
43.8 \\
143.058
\end{array}\right)
$$$$
\mathrm{f}\left(\operatorname{res}_{0}, \mathrm{res}_{1}\right)=2.238 \times 10^{4}
$$$$
\mathrm{x} 1+\operatorname{res}_{0}=243.8
$$$$
\mathrm{x} 2+\mathrm{res}_{1}=193.058
$$

Figure 3. Solving the inverse problem in MathCad

The equation constructed using data with numbers $2,3,7$ is as follows:

$$
\begin{aligned}
& g(x)=363036.5-275.49 \cdot x_{1}- \\
& -452.648 \cdot x_{2}
\end{aligned}
$$

Table 1. Simulation results in the first step of the algorithm

\begin{tabular}{|l|l|l|l|}
\hline $\begin{array}{c}\text { Calculation } \\
\text { number }\end{array}$ & Batch size & $\begin{array}{c}\text { Minimum } \\
\text { stock level }\end{array}$ & Average cost \\
\hline 1 & 200 & 50 & 281698.4 \\
\hline 2 & 250 & 50 & 271531.5 \\
\hline 3 & 250 & 100 & 248899.2 \\
\hline 4 & 150 & 50 & 300314.8 \\
\hline 5 & 150 & 100 & 276690.3 \\
\hline 6 & 150 & 0 & 419916.8 \\
\hline 7 & 200 & 100 & 262673.7 \\
\hline 8 & 200 & 0 & 419908.1 \\
\hline
\end{tabular}

The increments of the arguments according to the formula (8) are equal to $\left(x_{1 \min }^{*}=250, x_{2 \min }^{*}=100\right): \Delta_{1}=48$, $\Delta_{2}=79$. The values of the volume and minimum stock level are: $x_{1}=250+48=298, x_{2}=100+79=179$.

Table 2 presents the results of changing the obtained value in the given increment. For the values of the variables of the batch volume and the minimum stock level of 348 and 179 , respectively, the absolute deviation of the output value from its predetermined value is 292.61 . This value is less than 500 , so the operation of the algorithm ends.

Table 2. Simulation results in the fourth step of the algorithm

\begin{tabular}{|l|l|l|l|}
\hline $\begin{array}{c}\text { Calculation } \\
\text { number }\end{array}$ & Batch size & $\begin{array}{c}\text { Minimum } \\
\text { stock level }\end{array}$ & Average cost \\
\hline 1 & 298 & 179 & 214165.67 \\
\hline 2 & 348 & 179 & 199707.39 \\
\hline 3 & 248 & 179 & 208978.98 \\
\hline 4 & 298 & 229 & 179454.55 \\
\hline 5 & 298 & 129 & 220767.91 \\
\hline 6 & 348 & 229 & 186487.37 \\
\hline 7 & 348 & 129 & 228252.47 \\
\hline 8 & 248 & 229 & 194648.34 \\
\hline
\end{tabular}

Also, to solve the problem, direct search algorithms were considered. Table 3 presents the results of iterations when solving the problem by the method of exploratory search. The following values are the solution: the batch size is 325 , the minimum stock level is 200, the average cost is 199589.5, the absolute deviation of the average cost from the given value is 410.47 .

Table 3. Problem solving by the exploratory search method

\begin{tabular}{|l|l|l|l|}
\hline Increment size & $\begin{array}{l}\text { Batch } \\
\text { size }\end{array}$ & $\begin{array}{l}\text { Minimum } \\
\text { stock level }\end{array}$ & $\begin{array}{l}\text { Average } \\
\text { cost }\end{array}$ \\
\hline 50 & 250 & 100 & 248899.2 \\
\hline 50 & 300 & 150 & 217203.9 \\
\hline 50 & 300 & 200 & 198925.7 \\
\hline 25 & 325 & 200 & 199589.5 \\
\hline
\end{tabular}

Analyzing the results obtained, we may conclude that the approximation and the use of the inverse calculations with subsequent exploration search allows to find a solution to the inverse problem of the simulation modeling in fewer 
simulations as compared to the exploration search. As an example, Fig. 4 shows the number of simulations depending on a set step value, while using the adaptive search and solving the inverse problem with the help of the inverse calculations followed by the adaptive search (an algorithm based on the formation of the dependence function of an output value with respect to an input value).

There are also some other approaches how to solve to the inverse problem, among which we can note the regularization through the Manhattan distance, use of coefficients of relative priority of the indicators, minimax approach. In the first instance, the sum of the modules of changes of the arguments is minimized to achieve a set value of the function, in the second instance, the change is exercised in accordance with the set priority coefficients, in the third instance, the maximum of the absolute values of changes of the arguments is minimized.

Table 4 shows the results obtained using three methods of solving the inverse problem and an algorithm based on the formation of the dependence function of an output variable with respect to an input values.

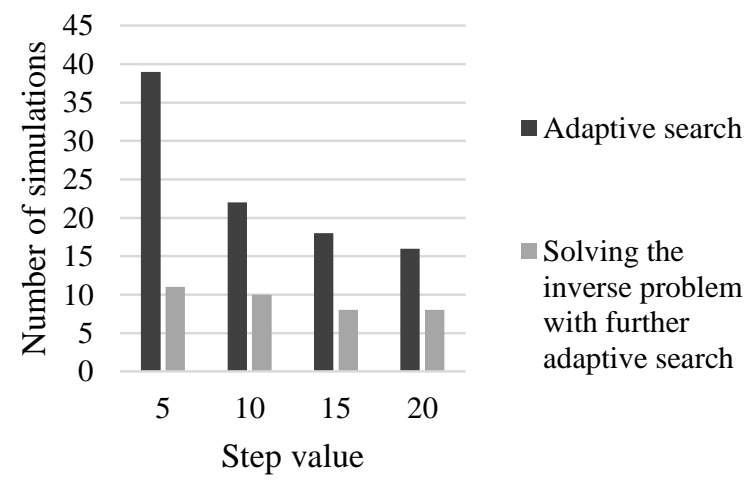

Figure 4. Number of simulations for the adaptive search and while using the inverse calculations followed by the adaptive search

Table 4. Inverse problem solving by the different approaches

\begin{tabular}{|l|l|l|l|}
\hline Regularization & $\begin{array}{l}\text { Batch } \\
\text { size }\end{array}$ & $\begin{array}{l}\text { Minimum } \\
\text { stock level }\end{array}$ & $\begin{array}{l}\text { Average } \\
\text { cost }\end{array}$ \\
\hline $\begin{array}{l}\text { Minimizing the sum } \\
\text { of squares of } \\
\text { changes of the } \\
\text { arguments }\end{array}$ & 244 & 193 & 204799.2 \\
\hline $\begin{array}{l}\text { Minimizing the sum } \\
\text { of changes of the } \\
\text { arguments modules }\end{array}$ & 200 & 209 & 225259.6 \\
\hline $\begin{array}{l}\text { Minimizing the } \\
\text { maximum from the } \\
\text { absolute values of } \\
\text { changes of the } \\
\text { arguments }\end{array}$ & 325 & 175 & 204896.6 \\
\hline
\end{tabular}

According to the values obtained in Table 4, it may be concluded that the use of regularization in the form of the sum of squares of changes of the arguments and the minimization of the maximum of the absolute values of the arguments ensure the best match to the set value $(200,000)$. However, minimizing the maximum of the absolute values of the arguments is a difficult task, while fairly simple iterative algorithms may be applied in case of minimizing the sum of squares of the arguments.

\section{CONCLUSION}

This paper considers the solution of inverse problems of simulation modelling. The use of direct search algorithms (research, adaptive, Hooke-Jeeves) is presented taking into account the specifics of the problem under consideration. The main disadvantage of these methods is the need to perform a large number of simulations with different values of the input variables, which can lead to high computational and time costs. Algorithms for solving the problem using approximation are proposed. The first algorithm consists in constructing a function of the dependence of the output variable on the input variables and solving the inverse problem by minimizing the increments of the arguments. The disadvantage of this method is the need to determine the dependence function and form a data set for function approximation. In the second algorithm a linear dependence function is iteratively constructed using the data set generated by changing the input variables in the given increments, and the inverse problem is solved by minimizing the increments of the arguments. The use of these methods also implies the subsequent use of direct search methods. The classical inventory management model with a threshold strategy is considered as an example. The use of approximation-based algorithms made it possible to solve the problem with fewer simulations as compared to direct search methods.

\section{References}

[1] R.C. Aster, B. Borchers, C.H. Thurber, Parameter Estimation and Inverse Problems, Elsevier, 2019, $404 \quad \mathrm{p}$ https://doi.org/10.1016/B978-0-12-804651-7.00015-8.

[2] D.L. Colton, Surveys on Solution Methods for Inverse Problems, Springer, Wien, 2000, 275 p. https://doi.org/10.1007/978-3-70916296-5.

[3] A.A. Shananin, "Inverse problems in economic measurements," Computational Mathematics and Mathematical Physics, vol. 58, issue 2, pp. 170-179, 2018. https://doi.org/10.1134/S0965542518020161.

[4] I. Ekeland, N. Djitte, "An inverse problem in the economy theory of demand," Annales de l'Institut Henri Poincare Non Linear Analysis, vol. 23, issue 2, pp. 269-281, 2006. https://doi.org/10.1016/j.anihpc.2005.10.001.

[5] S. I. Kabanihin, "Inverse problems of natural science and computer modeling," First-hand Science, vol. 49, issue 1, pp. 32-43, 2013. (in Russian)

[6] B.E. Odincov, Inverse Calculations in the Formation of Economic Decisions, Moscow: Finance and Statistics, 2004, 256 p. (in Russian)

[7] E. B. Gribanova, "Development of iterative algorithms for solving the inverse problem using inverse calculations," Eastern-European Journal of Enterprise Technologies, vol. 3, issue 4, pp. 27-34, 2020. https://doi.org/10.15587/1729-4061.2020.205048.

[8] F. Masoud, "A new efficient conjugate gradient method for unconstrained optimization," Journal of Computational and Applied Mathematics, vol. 300, pp. 207-216, 2016. https://doi.org/10.1016/j.cam.2015.12.035. 
[9] V. Cerda, J. L. Cerda, A. M. Idris, "Optimization using the gradient and simplex methods," Talanta, vol. 148, pp. 641-648, 2016. https://doi.org/10.1016/j.talanta.2015.05.061.

[10] S. Afandizadeh, M. Ameri, M. H. M. Moghaddam, "Introducing a modified gradient vector method for optimization of accident prediction non-linear functions," Applied Mathematical Modelling, vol. 190, pp. 5500-5506, 2011. https://doi.org/10.1016/j.apm.2011.03.053.

[11] M. Li, J. Bai, L. Li, X.Meng, Q. Liu, B. Chen, "A gradient-based aerostealth optimization design method for flying wing aircraft," Aerospace Science and Technology, vol. 92, pp. 156-169, 2019. https://doi.org/10.1016/j.ast.2019.05.067.

[12] C. Audet, "A survey on direct search methods for blackbox optimization and their applications," in: P.M. Pardalos, T.M. Rassias (Eds.), Mathematics without Boundaries, Springer, New York, 2014, pp. 31-56. https://doi.org/10.1007/978-1-4939-1124-0_2.

[13] T. G. Kolda, R. M. Lewis, V. T. Torczon, "Optimization by direct search: new perspectives on some classical and modern methods," SIAM review, vol. 45, issue 3, pp. 385-482, 2003. https://doi.org/10.1137/S003614450242889.

[14] F. Peng, K. Tang, G. Chen, X. Yao, "Population-based algorithm portfolios for numerical optimization," IEEE Transaction on Evolutionary Computation, issue 5, pp. 782-800, 2010. https://doi.org/10.1109/TEVC.2010.2040183.

[15] P. Vikhar, "Evolutionary algorithms: A critical review and its future prospects," Proceedings of the International Conference on Global Trends in Signal Processing, Information Computing and Communication, Jalgaon, India, December 22-24, 2016, pp. 261-265. https://doi.org/10.1109/ICGTSPICC.2016.7955308.

[16] J. Saldivar, C. Vairetti, C. Rodriguez, F. Daniel, F. Casati, R. Alarcon, "Analysis and improvement of business process models using spreadsheets," Information Systems, vol. 5, pp. 1-19, 2016. https://doi.org/10.1016/j.is.2015.10.012.

[17] A. Tiger, J. Loucks, C. Burns, "Spreadsheet-based supply chain simulation for teaching risk pooling combined with facility location,"
Independent Journal of Management \& Production, vol. 10, issue 6, pp. 1932-1951, 2019. https://doi.org/10.14807/ijmp.v10i6.960.

[18] B. Mileva Boshkoska, T. Damij, F. Jelenc, N. Damija, "Abdominal surgery process modeling framework for simulation using spreadsheets," Computer Methods and Programs in Biomedicine, vol. 121, issue $1, \quad$ pp. $1-13, \quad 2015$ https://doi.org/10.1016/j.cmpb.2015.05.001.

[19] R. Lewis, V. Torczon, M. Trosset, "Direct search methods: then and now," Journal of Computational and Applied Mathematics, vol. 124 issue 1-2, pp. 191-207, 2000. https://doi.org/10.1016/S03770427(00)00423-4.

[20] M. Gilli, D. Maringer, E. Schumann, Numerical Methods and Optimization in Finance, Academic Press, 2011, 256 p. https://doi.org/10.1016/B978-0-12-375662-6.00011-0.

[21] A. A. Mitsel, E. B. Gribanova, "Development of a system for simulating economic objects based on an object-oriented approach," Proceedings of Tomsk Polytechnic University, vol. 311, issue 6, pp. 11-15, 2007. (in Russian)

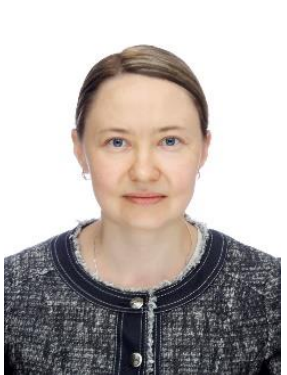

EKATERINA B. GRIBANOVA Candidate of Sciences (Engineering), Associate Professor of the Department of automated control systems of the Tomsk State University of Control Systems and Radioelectronics. Areas of scientific interests: economic modeling, simulation modeling, inverse problems, optimization methods, information systems and technologies. 